\title{
Actividad antifúngica in vitro de extractos crudos de Piper tuberculatum
}

\author{
In vitro antifungal activity of crude extracts of Piper tuberculatum
}

\author{
Zahyda G.F. Palacios ${ }^{1}$, Guillermo E. Delgado', Mario C. Moreno', Massuo J. Kato ${ }^{2}$ \\ y Consuelo Rojas ${ }^{* *}$
}

1 Facultad de Ciencias Biológicas, Universidad Nacional Pedro Ruiz Gallo, Ciudad Universitaria, Juan XXIII No 391, Lambayeque, Perú. Correspondecia Consuelo Rojas

2 Instituto de Química, Universidade de São Paulo, C.P. 26077, 05513-970, São Paulo, Brasil.

\begin{abstract}
Resumen
En la medicina tradicional Peruana Piper tuberculatum Jacq. (Piperaceae) es utilizado en humanos y animales domésticos como antiinflamatorio y desinfectante de heridas. Piper tuberculatum contiene las amidas isobutílicas, pirrolidina, dihidropiridona y piperidina. El objetivo de este trabajo fue investigar la actividad antifúngica de los extractos crudos de inflorescencias, hojas y tallos de plantas silvestres, obtenidos con $\mathrm{CH}_{2} \mathrm{Cl}_{2}: \mathrm{MeOH}$ (2:1), EtOH y decocción y de plantas in vitro obtenido con $\mathrm{CH}_{2} \mathrm{Cl}_{2}: \mathrm{MeOH}(2: 1)$. Los extractos crudos exhibieron actividad antifúngica sobre Trichophyton rubrum, Microsporum canis y M. gypseum. La concentración inhibitoria mínima (CIM) observada con los extractos $\mathrm{CH}_{2} \mathrm{Cl}_{2}: \mathrm{MeOH}(2: 1)$, EtOH y decocción, sobre Trichophyton rubrum, Microsporum canis y M. gypseum fue $0,1 \mathrm{mg} / \mathrm{mL}$ para inflorescencias y hojas, y 0,1 a $0,5 \mathrm{mg} / \mathrm{mL}$ para tallos. En plantas in vitro la inhibición en el crecimiento de T. rubrum y $M$. canis fue $100 \%$ en $0,5 \mathrm{mg} / \mathrm{mL}$ y para $M$. gypseum fue $95 \%$ en $1,5 \mathrm{mg} / \mathrm{mL}$ de concentración.
\end{abstract}

Palabras claves: cultivo de tejidos, extracto $\mathrm{CH}_{2} \mathrm{Cl}_{2}: \mathrm{MeOH}$, Microsporum canis, M. gypseum, Trichophyton rubrum.

\section{Abstract}

Piper tuberculatum Jacq. (Piperaceae) is used in traditional Peruvian medicine as anti-inflammatory and disinfectant of wounds in humans and domestic animals. This species contains amides bearing isobutyl, pyrrolidine, dihydropyridone and piperidine moieties. The aim of this work was to investigate antifungal activity of crude extracts from the spikes, leaves and stems of wild plants extracted with $\mathrm{CH}_{2} \mathrm{Cl}_{2}: \mathrm{MeOH}(2: 1), \mathrm{EtOH}$, decoction, and in vitro plants extracted with $\mathrm{CH}_{2} \mathrm{Cl}_{2}: \mathrm{MeOH}(2: 1)$. The crude extracts showed antifungal activity on Trichophyton rubrum, Mycosporum canis y M. gypseum. The minimum inhibitory concentration (MIC) observed with $\mathrm{CH}_{2} \mathrm{Cl}_{2}: \mathrm{MeOH}(2: 1)$, EtOH and decoctions extracts against T. rubrum, M. canis and $M$. gypseum was $0,1 \mathrm{mg} / \mathrm{mL}$ for spikes and leaves, and 0,1 to $0,5 \mathrm{mg} / \mathrm{mL}$ for stems. The inhibition of growth using in vitro plants on $T$. rubrum and $M$. canis was $100 \%$ in $0,5 \mathrm{mg} / \mathrm{mL}$, and $95 \%$ on $M$. gypseum $95 \%$ using $1,5 \mathrm{mg} / \mathrm{mL}$ of concentration.

Keywords: $\mathrm{CH}_{2} \mathrm{Cl}_{2}: \mathrm{MeOH}$ extract, Microsporum canis, M. gypseum, tissue culture, Trichophyton rubrum.

\section{Introducción}

Ha sido ampliamente reconocido que las plantas constituyen un inestimable reservorio de metabolitos secundarios biológicamente activos en el control de diversas enfermedades (Cos et al. 2008; Gibbons 2008) y las provocadas por hongos en particular (Grayer \& Harborne 1994; Osburn 1996). Los efectos secundarios adversos de algunas drogas, la resistencia frecuentemente inducida y los elevados costos en el tratamiento (Davicino et al. 2007), han estimulado y orientado muy intensamente la investigación en la búsqueda y utilización de sustancias puras o extractos crudos de plantas en el control de diversas enfermedades fúngicas (Dabur et al. 2004; Fenner et al. 2005; Zhang et al. 2005). Así tenemos que el extracto crudo de varias especies utilizadas en la medicina tradicional peruana como Cestrum auriculatum, Iryanthera lancifolia y Wigandia urens, entre otras, mostraron actividad inhibitoria sobre Trycophyton mentagrophytes y Microsporum gypseum, entre otros patógenos (Rojas et al. 2003); el aceite esencial, constituido por varios compuestos como $\beta$-Eudesmol, espatulenol e isocariofilene, entre otros, y extractos crudos de Cordia curassavica, mostraron una significativa actividad antibacteriana y antifúngica (Hernández et al. 2007) y el aceite esencial de hojas de Minthostachys mollis, donde fueron identificados los terpenoides pulegona, mentona, limoneno y mirceno, mostró una significativa actividad contra Candida albicans, T. mentagrophytes y Microsporum canis (Cano et al. 2008).

En especies de Piper como P. tuberculatum, P. hispidum y $P$. arboreum, han sido aisladas las amidas piperamina, piplartina, piperina, 5,6-hidropiperlonguminina, arboreumina, fagaramida, entre otras (Navickiene at al. 2000; Silva et al. 2002) y en $P$. crassinervium, fueron aisladas varias flavononas e hidroquinonas preniladas (Danelutte et al. 2003), todas ellas con actividad antifúngica sobre Cladosporium cladosporioides y C. sphaerospermum en ensayos por autografía directa; en $P$. regnellii el extracto crudo y las neolignanas eupomatenoide- 3 y eupomatenoide- 5 mostraron una fuerte actividad sobre T. mentagrophytes, T. rubrum, $M$. canis y M. gypseum (Koroishi et al. 2008) y varias especies de Candida (Pessini et al. 2005).

Estudios químicos realizados en especies de la familia Piperaceae del Brasil han revelado la ocurrencia de diversos productos naturales fisiológicamente activos tales como alcaloides, amidas, pironas, dihidrochalconas, flavonoides, fenilpropanoides, lignanos y neolignanos (Orjala et al. 1994; Martins et al. 2000; Danelutte et al. 2003). Varias amidas isobutílicas, pirrolidina, dihidroxipiridona y piperidina (Alécio et al. 1998; Navickiene et al. 2000; Silva et al. 2002) han sido determinadas. Estas amidas han generado interés debido a sus potentes propiedades insecticidas y antifúngicas (Miyakado et al. 1989; Alécio et al. 1998; Navickiene et al. 2000; Silva et al. 2002; Soberón et al. 2006).

En trabajos previos (Navickiene et al. 1999; 2000), se ha reportado el aislamiento, la estructura química y la actividad antifúngica de varias amidas como la piplartina, piperamina, piperina, pelitorina, entre otras, aisladas de semillas y hojas de Piper tuberculatum. Los ensayos se realizaron con los hongos Cladosporium sphaerospermum (Navickiene et al. 2000) y $C$. sphaerospermum y C. cladosporioides (Silva et al. 2002), utilizando el método de bioautografía directa (Homans \& Fuchs 1970). 
Por otro lado, la correcta identificación taxonómica de $P$. tuberculatum Jacq. (Trelease 1936; Yuncker 1973) ha sido motivo de cierta controversia puesto que, recientemente, ha sido sinonimizada como Piper arboreum subsp. tuberculatum (Jacq.) Tebbs stat. nov. (Tebbs 1989), en base a algunas diferencias morfológicas con $P$. arboreum subsp. arboreum Aublet; sin embargo, estudios fitoquímicos comparativos han revelado que amidas extraídas de hojas de $P$. arboreum son diferentes a las extraídas de $P$. tuberculatum (Navickiene et al., 2002), por lo que sugerimos que $P$. tuberculatum debe ser considerada una especie distinta, con una amplia distribución desde México a Paraguay, reportándose para el Perú en las regiones de Amazonas, Huánuco, Lambayeque, Loreto, San Martín, Tumbes y Ucayali (Brako \& Zarucchi, 1993).

El objetivo del presente trabajo fue evaluar la actividad antifúngica de los extractos crudos de diclormetano-metanol $\left(\mathrm{CH}_{2} \mathrm{Cl}_{2}: \mathrm{MeOH}\right)(2: 1)$, etanol $(\mathrm{EtOH})$ y decocciones, de inflorescencias, hojas y tallos de plantas silvestres y plantas in vitro de Piper tuberculatum ("matico" o "cordoncillo") sobre Trichophyton rubrum, Microsporum canis y M. gypseum.

\section{Materiales y métodos}

Material vegetal. Inflorescencias con semillas maduras, hojas y tallos de $P$. tuberculatum Jacq. fueron colectadas en el mes de noviembre del 2003 en el Río Cumbil (Lambayeque, Perú); la especie fue identificada por el Dr. Guillermo E. Delgado de la Universidad Nacional Pedro Ruiz Gallo (UNPRG) de Lambayeque, Perú. Una muestra herborizada fue depositada en el Herbarium Pedro Ruiz Gallo, Lambayeque. Las plantas in vitro fueron obtenidas de semillas germinadas in vitro y micropropagadas en tal condición por cultivo de ápices y segmentos nodales.

Microorganismos. Los microorganismos utilizados en el ensayo antifúngico, T. rubrum, $M$. canis y $M$. gypseum fueron proporcionados por el Laboratorio de Micología de la Universidad Nacional Mayor de San Marcos, Lima, Laboratorio de Micología de la Región II de Salud, MINSA y Laboratorio de Micología del Instituto Nacional de Salud del Perú. Las cepas fueron conservadas en medio de cultivo Agar Sabouraud Glucosado (ASG) $2 \%$ a $4{ }^{\circ} \mathrm{C}$ de temperatura y repicadas periódicamente.

Obtención de los extractos. En el caso de plantas silvestres, $45 \mathrm{~g}$ de inflorescencias, hojas y tallos, respectivamente, fueron secados en estufa a $40{ }^{\circ} \mathrm{C}$ durante una semana, reducidos a polvo fino y sometidos a extracción por 3 veces consecutivas durante $24 \mathrm{~h}$ y a temperatura ambiente; los solventes de extracción utilizados fueron diclorometano-metanol $\left(\mathrm{CH}_{2} \mathrm{Cl}_{2}: \mathrm{MeOH}\right)$ (2:1) y alcohol etílico (EtOH). En el caso de plantas micropropagadas in vitro se tomó $9 \mathrm{~g}$ de muestra la que fue sometida al mismo procedimiento anteriormente descrito, pero utilizando únicamente $\mathrm{CH}_{2} \mathrm{Cl}_{2}: \mathrm{MeOH}$ (2:1), como solvente de extracción; luego el extracto se separó por evaporación a presión reducida y $45^{\circ} \mathrm{C}$ de temperatura. En las decocciones se utilizaron $10 \mathrm{~g}$ de polvo seco de inflorescencias, hojas y tallos de plantas silvestres, suplementándose $100 \mathrm{ml}$ de agua destilada hirviendo y manteniéndose en ebullición durante $10 \mathrm{~min}$; luego fueron filtrados en condiciones de asepsia total.

Preparación y estandarización del inóculo. Con la finalidad de obtener un número adecuado de esporas las especies en estudio fueron sembradas en medio de cultivo ASG 2\% y una vez alcanzada la esporulación fueron lavadas con solución salina fisiológica esterilizada de manera que el sobrenadante pudiera estandarizarse por turbidometría al tubo $\mathrm{N}^{\circ} 0,5$ del nefelómetro de Mc Farland, que corresponde a una concentración equivalente a $1 \times 10^{8}$ gérmenes $/ \mathrm{mL}$, para conseguir en todos los ensayos una biomasa uniforme.

Actividad antifúngica. Los extractos obtenidos con $\mathrm{CH}_{2} \mathrm{Cl}_{2}: \mathrm{MeOH}(2: 1)$ y EtOH y por decocción, fueron preparados en cuatro concentraciones diferentes $(0,1 ; 0,5 ; 1,0$ y $1,5 \mathrm{mg} /$ $\mathrm{mL}$ ) de acuerdo al método de dilución en agar (Navarro-García et al. 2003). En tal sentido, la solución stock fue preparada en proporción 10:1 entre la masa del extracto y la solución $\mathrm{MeOH}$ agua, obteniendo una concentración equivalente a $10 \mathrm{mg} / \mathrm{mL}$. Para el ensayo de actividad antifúngica se tomó 0,$1 ; 0,5 ; 1,0$ y $1,5 \mathrm{~mL}$ de esta solución stock suplementándose con 9,9; 9,5; 9,0 y 8,5 mL de medio de cultivo ASG, respectivamente, que luego fue aplicada a placas de Petri esterilizadas de 100x15 mm. Las placas de Petri fueron inoculadas con suspensión de esporas estandarizada a $0,1 \mathrm{~mL}$, diseminadas con espátula de Drigalski e incubadas por 7-10 días en oscuridad en cámara húmeda a $25^{\circ} \mathrm{C}$ de temperatura. Cada tratamiento fue repetido 3 veces y el experimento general dos veces. Como control negativo se emplearon placas de Petri sin extractos y para descartar el efecto de la solución $\mathrm{MeOH}$-agua el tratamiento control fue suplementado con 1,5 mL de dicha solución.

Se estableció la actividad antifúngica determinando la concentración inhibitoria mínima (CIM), definida como la concentración más baja de extracto capaz de inhibir el crecimiento visible en el agar (Navarro-García et al., 2003).

Análisis estadístico. Se utilizó el diseño experimental con estímulo creciente donde los grupos experimentales fueron las especies fúngicas y el estímulo creciente las concentraciones de extractos. Los datos expresados en porcentajes de crecimiento de las especies fúngicas fueron transformados a $\sqrt{x}_{\mathrm{x}}+1$ para una distribución normal de los errores experimentales. Se realizaron análisis de varianza para cada especie fúngica así como las pruebas de comparaciones múltiples de Tukey con un nivel de significación de $0,05 \%$ aplicando un diseño con arreglo factorial $4 \times 3$ ( 4 concentraciones, 3 estructuras vegetales) y 3 repeticiones para cada tipo de extracto.

Micropropagación de plantas. El cultivo in vitro fue iniciado a partir de explantes obtenidos de semillas asépticas. Las semillas (50 por frasco) fueron desinfestadas con etanol $70 \%(\mathrm{v} / \mathrm{v})$ durante $1 \mathrm{~min}$ seguido con hipoclorito de sodio $2,5 \%(\mathrm{w} / \mathrm{v})$ durante $20 \mathrm{~min}$. Luego fueron lavadas por tres veces consecutivas con agua destilada esterilizada. Las semillas flotantes fueron descartadas y alrededor de 3-10 de estas fueron transferidas a tubos de ensayo de $120 \times 16$ mm conteniendo el medio de cultivo con las sales minerales MS (Murashige \& Skoog 1962), sacarosa 2\% y agar $0,8 \%$. Ápices caulinares y segmentos nodales, de un $\mathrm{cm}$ de longitud conteniendo una yema lateral, tomados de plantas germinadas in vitro de tres meses de edad, fueron usados como fuente de explantes. Las sales minerales MS, suplementadas con varias concentraciones de ácido indol-3-acético (AIA), ácido giberélico $\left(\mathrm{AG}_{3}\right)$, 6-benzilaminopurina (BAP) y sacarosa 3\%, fueron utilizadas en el medio de cultivo inicial y el mantenimiento de los cultivos se realizó cada 6 meses transfiriéndolos a medio de cultivo fresco de la misma formulación. En todos los cultivos, las sales minerales MS fueron suplementadas con m-inositol 100 mg/L y tiamina.HCl $1 \mathrm{mg} / \mathrm{L}$, ajustándose el pH 
Tabla 1. Inhibición en el crecimiento de Trichophyton rubrum por efecto de varias concentraciones de extractos de inflorescencias, hojas y tallos de Piper tuberculatum obtenidos con $\mathrm{CH}_{2} \mathrm{Cl}_{2}: \mathrm{MeOH}$ (2:1), EtOH y decocción.

\begin{tabular}{|c|c|c|c|c|}
\hline \multirow{2}{*}{$\begin{array}{l}\text { Partes de } \\
\text { la planta } \\
\text { utilizadas }\end{array}$} & \multirow{2}{*}{$\begin{array}{l}\text { Concentración } \\
(\mathrm{mg} / \mathrm{mL})\end{array}$} & \multicolumn{3}{|c|}{ Inhibición (\%) } \\
\hline & & $\begin{array}{l}\mathrm{CH}_{2} \mathrm{Cl}_{2}: \\
\mathrm{MeOH}^{\mathrm{a}}\end{array}$ & $\mathrm{EtOH}^{\mathrm{b}}$ & Decocción \\
\hline \multirow{4}{*}{ Inflorescencia } & 0,1 & 100 & 88,3 & 85 \\
\hline & 0,5 & 100 & 100 & 100 \\
\hline & 1,0 & 100 & 100 & 100 \\
\hline & 1,5 & 100 & 100 & 100 \\
\hline \multirow{4}{*}{ Hojas } & 0,1 & 63,3 & 53,3 & 5 \\
\hline & 0,5 & 100 & 100 & 63,3 \\
\hline & 1,0 & 100 & 100 & 96,7 \\
\hline & 1,5 & 100 & 100 & 100 \\
\hline \multirow{4}{*}{ Tallos } & 0,1 & 8,3 & 10 & 5 \\
\hline & 0,5 & 100 & 20 & 6,7 \\
\hline & 1,0 & 100 & 61,7 & 6,7 \\
\hline & 1,5 & 100 & 98,3 & 6,7 \\
\hline
\end{tabular}

a: $\mathrm{CH}_{2} \mathrm{Cl}_{2}=$ diclorometano, $\mathrm{MeOH}=$ metanol

b: $\mathrm{EtOH}=$ etanol

$5,7 \pm 0,1$, con $\mathrm{NaOH}$ o $\mathrm{HCl} 0,5 \mathrm{~N}$, gelificándose con "Phytagel" $0,2 \%$ y dispensándose en tubos de ensayo de $150 \times 25 \mathrm{~mm}$ antes de la esterilización en autoclave a $121{ }^{\circ} \mathrm{C}$ de temperatura y 15 lbs/pulg ${ }^{2}$ de presión durante 20 min. Todos los cultivos fueron incubados en $24-28^{\circ} \mathrm{C}$, fotoperiodo de $16-8 \mathrm{~h}$ de luz/oscuridad proporcionada por tubos de fluorescentes de color blanco con una irradiancia de $5 \mu \mathrm{mol} \mathrm{m} \mathrm{m}^{-2} \mathrm{~s}^{-1}$, para la germinación de semillas y $30 \mu \mathrm{mol} \mathrm{m} \mathrm{m}^{-2} \mathrm{~s}^{-1}$ para la micropropagación.

\section{Resultados y discusión}

El rendimiento de extractos de $45 \mathrm{~g}$ de materia seca con $\mathrm{CH}_{2} \mathrm{Cl}_{2}: \mathrm{MeOH}(2: 1)$ de inflorescencias, hojas y tallos, de plantas de campo de $P$. tuberculatum fue 2,7 (6,0\%); 2,0 (4,4\%) y 0,7 $(1,6 \%) \mathrm{g}$, respectivamente, y con $\mathrm{EtOH}$ fue 5,3 (11,8\%); 2,3 $(5,0 \%)$ y $0,9(2,1 \%) \mathrm{g}$, respectivamente, en tanto que el rendimiento de $9,0 \mathrm{~g}$ de extracto de plantas micropropagadas in vitro con $\mathrm{CH}_{2} \mathrm{Cl}_{2}: \mathrm{MeOH}(2: 1)$ fue $0,6 \mathrm{~g}(6,3 \%)$.

En eI caso de T. rubrum los extractos de $\mathrm{CH}_{2} \mathrm{Cl}_{2}: \mathrm{MeOH}$ (2:1) de todas las partes de las plantas utilizadas (inflorescencias

Tabla 2. Inhibición en el crecimiento de Microsporum canis por efecto de varias concentraciones de extractos de inflorescencias, hojas y tallos de Piper tuberculatum obtenidos con $\mathrm{CH}_{2} \mathrm{Cl}_{2}: \mathrm{MeOH}(2: 1)$, EtOH y decocción.

\begin{tabular}{|c|c|c|c|c|}
\hline \multirow{2}{*}{$\begin{array}{l}\text { Partes de } \\
\text { la planta } \\
\text { utilizadas }\end{array}$} & \multirow{2}{*}{$\begin{array}{l}\text { Concentración } \\
(\mathrm{mg} / \mathrm{mL})\end{array}$} & \multicolumn{3}{|c|}{ Inhibición (\%) } \\
\hline & & $\begin{array}{l}\mathrm{CH}_{2} \mathrm{Cl}_{2}: \\
\mathrm{MeOH}^{\mathrm{a}}\end{array}$ & $\mathrm{EtOH}^{\mathrm{b}}$ & Decocción \\
\hline \multirow{4}{*}{ Inflorescencia } & 0,1 & 91,7 & 100 & 91,7 \\
\hline & 0,5 & 100 & 100 & 100 \\
\hline & 1,0 & 100 & 100 & 100 \\
\hline & 1,5 & 100 & 100 & 100 \\
\hline \multirow{4}{*}{ Hojas } & 0,1 & 68,3 & 70 & 25 \\
\hline & 0,5 & 100 & 100 & 88,3 \\
\hline & 1,0 & 100 & 100 & 96,7 \\
\hline & 1,5 & 100 & 100 & 100 \\
\hline \multirow{4}{*}{ Tallos } & 0,1 & 28,3 & 55 & 30 \\
\hline & 0,5 & 98,3 & 99,3 & 90 \\
\hline & 1,0 & 100 & 100 & 96,7 \\
\hline & 1,5 & 100 & 100 & 100 \\
\hline
\end{tabular}

a: $\mathrm{CH}_{2} \mathrm{Cl}_{2}=$ diclorometano, $\mathrm{MeOH}=$ metanol

b: $\mathrm{EtOH}^{2}=$ etanol
Tabla 3. Inhibición en el crecimiento de Microsporum gypseum por efecto de varias concentraciones de extractos de inflorescencias, hojas y tallos de Piper tuberculatum obtenidos con $\mathrm{CH}_{2} \mathrm{Cl}_{2}: \mathrm{MeOH}$ (2:1), EtOH y decocción.

\begin{tabular}{|c|c|c|c|c|}
\hline \multirow{2}{*}{$\begin{array}{l}\text { Partes de } \\
\text { la planta } \\
\text { utilizadas }\end{array}$} & \multirow{2}{*}{$\begin{array}{l}\text { Concentración } \\
(\mathrm{mg} / \mathrm{mL})\end{array}$} & \multicolumn{3}{|c|}{ Inhibición (\%) } \\
\hline & & $\begin{array}{l}\mathrm{CH}_{2} \mathrm{Cl}_{2}: \\
\mathrm{MeOH}^{\mathrm{a}}\end{array}$ & $\mathrm{EtOH}^{\mathrm{b}}$ & Decocción \\
\hline \multirow{4}{*}{ Inflorescencia } & 0,1 & 100 & 100 & 100 \\
\hline & 0,5 & 100 & 100 & 100 \\
\hline & 1,0 & 100 & 100 & 100 \\
\hline & 1,5 & 100 & 100 & 100 \\
\hline \multirow{4}{*}{ Hojas } & 0,1 & 38,3 & 76,7 & 6,7 \\
\hline & 0,5 & 100 & 100 & 95 \\
\hline & 1,0 & 100 & 100 & 100 \\
\hline & 1,5 & 100 & 100 & 100 \\
\hline \multirow{4}{*}{ Tallos } & 0,1 & 18,3 & 8,3 & 5 \\
\hline & 0,5 & 100 & 91,7 & 13,3 \\
\hline & 1,0 & 100 & 100 & 13,3 \\
\hline & 1,5 & 100 & 100 & 15 \\
\hline
\end{tabular}

a: $\mathrm{CH}_{2} \mathrm{Cl}_{2}=$ diclorometano, $\mathrm{MeOH}=$ metanol

b: $\mathrm{EtOH}^{2}=$ etanol

$0,1-1,5 \mathrm{mg} / \mathrm{mL}$ y hojas y tallos $0,5-1,0 \mathrm{mg} / \mathrm{mL}$ ), fueron altamente activos, inhibiendo $100 \%$ el crecimiento del hongo; un efecto inhibitorio similar fue, también, observado con extractos de EtOH de inflorescencias y hojas $(0,5-1,5 \mathrm{mg} / \mathrm{mL})$, en tanto que únicamente las decocciones de inflorescencias $(0,5-1,5$ $\mathrm{mg} / \mathrm{mL})$ y hojas $(1,5 \mathrm{mg} / \mathrm{mL})$ permitieron alcanzar $100 \%$ de inhibición en el crecimiento del hongo (Tabla 1).

Referente a $M$. canis, solamente las más altas concentraciones de extractos de $\mathrm{CH}_{2} \mathrm{Cl}_{2}: \mathrm{MeOH}(2: 1)$ de inflorescencias y hojas $(0,5-1,5 \mathrm{mg} / \mathrm{mL})$ y tallos $(1,0-1,5 \mathrm{mg} / \mathrm{mL})$ mostraron $100 \%$ de inhibición en el crecimiento del hongo; un efecto inhibitorio similar fue observado con extractos de $\mathrm{EtOH}$ de inflorescencias $(0,1-1,5 \mathrm{mg} / \mathrm{mL})$, hojas $(0,5-1,5 \mathrm{mg} / \mathrm{mL})$ y tallos $(1,0-1,5 \mathrm{mg} / \mathrm{mL})$, en tanto que únicamente las decocciones de inflorescencias $(0,5-1,5 \mathrm{mg} / \mathrm{mL})$, hojas y tallos $(1,5 \mathrm{mg} / \mathrm{mL})$ permitieron alcanzar $100 \%$ de inhibición en el crecimiento del hongo (Tabla 2).

Sobre M. gypseum, solamente las mayores concentraciones de extractos de $\mathrm{CH}_{2} \mathrm{Cl}_{2}: \mathrm{MeOH}$ (2:1) de inflorescencias (0,1-1,5 $\mathrm{mg} / \mathrm{mL})$, hojas $(0,5-1,5 \mathrm{mg} / \mathrm{mL})$ y tallos $(0,5-1,5 \mathrm{mg} / \mathrm{mL})$, mostraron $100 \%$ de inhibición en el crecimiento del hongo; un efecto inhibitorio similar fue observado con extractos de EtOH de inflorescencias $(0,1-1,5 \mathrm{mg} / \mathrm{mL})$, hojas $(0,5-1,5 \mathrm{mg}$ / $\mathrm{mL})$ y tallos $(1,0-1,5 \mathrm{mg} / \mathrm{mL})$, en tanto que únicamente las decocciones de inflorescencias $(0,1-1,5 \mathrm{mg} / \mathrm{mL})$, hojas y tallos $(1,5 \mathrm{mg} / \mathrm{mL})$ permitieron alcanzar $100 \%$ de inhibición en el crecimiento del hongo (Tabla 3). La concentración inhibitoria mínima (CIM), observada con los tres tipos de extractos ensayados, contra T. rubrum, $M$. canis y $M$. gypseum, fue $0,1 \mathrm{mg} /$ $\mathrm{mL}$, para inflorescencias y hojas, y $0,1-0,5 \mathrm{mg} / \mathrm{mL}$ para tallos (Tabla 4). En los tratamientos control el crecimiento de los dermatofitos fue $100 \%$.

Todas las especies de dermatofitos estudiados resultaron altamente susceptibles a la actividad inhibitoria de los tipos de extractos ensayados. Los extractos de $\mathrm{CH}_{2} \mathrm{Cl}_{2}: \mathrm{MEOH}(2: 1)$ exhibieron una mayor actividad antifúngica que los extractos de EtOH y estos que los obtenidos por decocción. Asimismo, los extractos de inflorescencias fueron más eficientes que los de hojas y estos que los obtenidos de tallos, en tanto que las 
Tabla 4. Concentración inhibitoria mínima (CIM) de extractos $\mathrm{CH}_{2} \mathrm{Cl}_{2}: \mathrm{MeOH}^{\mathrm{a}}(2: 1), \mathrm{EtOH}^{\mathrm{b}}$ y decocción de Piper tuberculatum sobre Trichophyton rubrum, Microsporum canis y Microsporum gypseum. Valores en $\mathrm{mg} / \mathrm{mL}$.

\begin{tabular}{lccc}
\hline & $\mathrm{CH}_{2} \mathrm{Cl}_{2}: \mathrm{MeOH}$ & $\mathrm{EtOH}$ & Decocción \\
\cline { 2 - 4 } Inflorescencias & \multicolumn{3}{c}{ Trichophyton rubrum } \\
\cline { 2 - 4 } Hojas & 0,1 & 0,1 & 0,1 \\
Tallos & 0,1 & 0,1 & 0,1 \\
& 0,5 & 0,5 & - \\
\hline \multirow{3}{*}{ Inflorescencias } & 0,1 & 0,1 & 0,1 \\
Hojas & 0,1 & 0,1 & 0,1 \\
Tallos & 0,1 & 0,1 & 0,1 \\
\hline \multirow{3}{*}{ Inflorescencias } & \multicolumn{3}{c}{ Microsporum gypseum } \\
Hojas & 0,1 & 0,1 & 0,1 \\
Tallos & 0,1 & 0,1 & 0,5 \\
\hline
\end{tabular}

a: $\mathrm{CH}_{2} \mathrm{Cl}_{2}=$ diclorometano, $\mathrm{MeOH}=$ metanol

b: $\mathrm{EtOH}=$ etanol

Tabla 5. Prueba de significación de Tukey para Trichophyton rubrum, Microsporum canis y Microsporum gypseum respecto a los factores concentración del extracto $\left[\mathrm{CH}_{2} \mathrm{Cl}_{2}: \mathrm{MeOH}\right.$ (2:1), EtOH y decocción] y partes de la planta (inflorescencias, hojas y tallos) de Piper tuberculatum ${ }^{\mathrm{a}, \mathrm{b}, \mathrm{c}}$.

\begin{tabular}{|c|c|c|c|}
\hline \multirow{2}{*}{$\begin{array}{l}\text { Concentración } \\
(\mathrm{mg} / \mathrm{mL})\end{array}$} & \multicolumn{3}{|c|}{ Inhibición (\%) } \\
\hline & $\mathrm{CH}_{2} \mathrm{Cl}_{2}: \mathrm{MeOH}$ & $\mathrm{EtOH}$ & Decocción \\
\hline & \multicolumn{3}{|c|}{ Trichophyton rubrum } \\
\hline 1,5 & $100,0 \mathrm{a}$ & $99,4 \mathrm{a}$ & 68,9 a \\
\hline 1,0 & $100,0 \mathrm{a}$ & $87,2 \mathrm{~b}$ & $67,8 \mathrm{a}$ \\
\hline 0,5 & $100,0 \mathrm{a}$ & $73,3 \mathrm{c}$ & $56,7 \mathrm{~b}$ \\
\hline \multirow[t]{2}{*}{0,1} & $57,2 \mathrm{~b}$ & $50,6 \mathrm{~d}$ & $31,7 \mathrm{c}$ \\
\hline & \multicolumn{3}{|c|}{ Microsporum canis } \\
\hline 1,5 & $100,0 \mathrm{a}$ & $100,0 \mathrm{a}$ & $100,0 \mathrm{a}$ \\
\hline 1,0 & $100,0 \mathrm{a}$ & $100,0 \mathrm{a}$ & $97,2 \mathrm{a}$ \\
\hline 0,5 & $99,4 \mathrm{a}$ & 99,4 a & $92,8 \mathrm{~b}$ \\
\hline \multirow[t]{2}{*}{0,1} & $62,8 \mathrm{~b}$ & $75,0 \mathrm{~b}$ & $48,9 \mathrm{c}$ \\
\hline & \multicolumn{3}{|c|}{ Microsporum gypseum } \\
\hline 1,5 & $100,0 \mathrm{a}$ & $100,0 \mathrm{a}$ & $71,7 \mathrm{a}$ \\
\hline 1,0 & $100,0 \mathrm{a}$ & $100,0 \mathrm{a}$ & $69,4 \mathrm{a}$ \\
\hline 0,5 & $100,0 \mathrm{a}$ & $97,2 \mathrm{a}$ & $42,2 \mathrm{~b}$ \\
\hline 0,1 & $52,2 \mathrm{~b}$ & $61,7 \mathrm{~b}$ & $37,2 \mathrm{~b}$ \\
\hline \multirow[t]{2}{*}{ Partes - planta } & $\mathrm{CH}_{2} \mathrm{Cl}_{2}: \mathrm{MeOH}$ & $\mathrm{EtOH}$ & Decocción \\
\hline & \multicolumn{3}{|c|}{ Trichophyton rubrum } \\
\hline Inflorescencia & $100,0 \mathrm{a}$ & 97,0 a & $96,2 \mathrm{a}$ \\
\hline Hojas & $90,8 \mathrm{~b}$ & $88,3 \mathrm{~b}$ & $66,2 \mathrm{~b}$ \\
\hline \multirow[t]{2}{*}{ Tallos } & $77,0 \mathrm{c}$ & $47,0 \mathrm{c}$ & $6,2 \mathrm{c}$ \\
\hline & \multicolumn{3}{|c|}{ Microsporum canis } \\
\hline Inflorescencia & $100,0 \mathrm{a}$ & $97,9 \mathrm{a}$ & $97,9 \mathrm{a}$ \\
\hline Hojas & $92,5 \mathrm{~b}$ & $92,0 \mathrm{~b}$ & $78,7 \mathrm{~b}$ \\
\hline \multirow[t]{2}{*}{ Tallos } & $88,3 \mathrm{c}$ & $81,7 \mathrm{c}$ & $77,5 \mathrm{~b}$ \\
\hline & \multicolumn{3}{|c|}{ Microsporum gypseum } \\
\hline Inflorescencia & $100,0 \mathrm{a}$ & $100,0 \mathrm{a}$ & $100,0 \mathrm{a}$ \\
\hline Hojas & $84,6 \mathrm{~b}$ & $94,2 \mathrm{~b}$ & $53,3 \mathrm{~b}$ \\
\hline Tallos & $79,6 \mathrm{c}$ & $75,0 \mathrm{c}$ & $11,3 \mathrm{c}$ \\
\hline
\end{tabular}

a: Valores con la misma letra son significativamente iguales.

b: $\mathrm{CH}_{2} \mathrm{Cl}_{2}=$ diclorometano, $\mathrm{MeOH}=$ metanol

c: $\mathrm{EtOH}=$ etanol concentraciones de 0,5 a $1,5 \mathrm{mg} / \mathrm{mL}$ mostraron, también, la más alta actividad biológica contra T. rubrum, $M$. canis y $M$. gypseum (Tabla 5).

Varios autores han reportado la actividad antifúngica de diversos extractos de plantas sobre diversos hongos, resultando relativamente recientes los trabajos referidos a los dermatofitos Trichophyton y Microsporum. Así tenemos que para T. mentagrophytes la zona de inhibición en el crecimiento fue entre 19-31 $\mathrm{mm}$ con $25 \mathrm{mg} / \mathrm{mL}$ del extracto etanólico de hojas de Cestrum auriculatum, Iryanthera lancifolia, Ophryosporus peruvianus y Wigandia urens y para $M$. gypseum fue $21 \mathrm{~mm}$ con $C$. auriculatum (Rojas et al. 2003). Diferentes especies de Trichophyton, entre ellas, T. rubrum resultaron con una inhibición en el crecimiento entre $+\mathrm{a}+++$, con una escala máxima de ++++ , utilizando extractos etanólico y acuoso de Solanum nigrum, Aloe vera y Allium sativum en diluciones de 1:10 a 1:500 de $100 \mathrm{mg} / \mathrm{mL}$ (Shamim et al. 2004). Asimismo, $0,1 \mathrm{~mL}$ ( 50 y $100 \%$ de concentración) y 5-10 $\mu \mathrm{L} / 10 \mathrm{~mL}$, de aceite esencial de Minthostachys mollis, con los métodos de agar en difusión y dilución en tubo, respectivamente, inhibieron $100 \%$ el crecimiento de T. mentagrophytes, T. tonsurans y $M$. canis (Cano et al. 2008). También, el extracto etanólico de Piper betle, Alpinia galanga y Allium ascalonicum mostraron actividad antidermatofítica sobre T. mentagrophytes, $M$. canis y $M$. gypseum, destacando $P$. betle con una $\mathrm{CI}_{50}$ entre 110,4-119,0 $\mu \mathrm{g} / \mathrm{mL}$ (Trakranrungsie et al. 2008) y el extracto crudo hidroalcohólico y las neolignanas eupomatenoide-3 y eupomatenoide-5, de Piper regnellii, mostraron actividad anidermatofítica sobre T. mentagrophytes, T. rubrum, $M$. canis con $\mathrm{CL}_{90}$ de $15,6 \mu \mathrm{g} / \mathrm{mL}$ y para $M$. gypseum con $\mathrm{CL}_{90}$ de $62,5 \mu \mathrm{g} /$ $\mathrm{mL}$ (Koroishi et al. 2008). Todos estos trabajos nos indican que el extracto crudo de varias especies vegetales exhibe una significativa actividad antifúngica sobre diversos dermatofitos, variando significativamente sus efectos en función de las concentraciones utilizadas, destacando las especies $P$. betle y $P$. regnellii, de origen asiático y americano, respectivamente.

Otros trabajos han reportado la ocurrencia de varias especies de Microsporum en animales en cautiverio (Levy et al. 2006) y silvestres (Araújo et al. 2008) pero sin proponer medidas de control. Por otro lado, la literatura consultada reporta apenas el trabajo de Davicino et al. (2007) utilizando decocciones de algunas plantas medicinales de Argentina mostrando actividad antifúngica sobre algunos microorganismos como Candida albicans y Saccharomyces cereviceae. En nuestro trabajo, las decocciones de inflorescencias, y en menor proporción las de hojas, resultaron muy efectivas en la inhibición del crecimiento de las tres especies de dermatofitos ensayadas. Con certeza la obtención de extractos por decocción así como con EtOH abre la posibilidad de su utilización amplia por parte de las poblaciones rurales; en este último caso utilizando solventes caseros como el "yonque" o "cańazo", aguardiente obtenido artesanalmente de la caña de azúcar.

En relación a los extractos de otras especies de Piperaceae (Piper y Peperomia), trabajos previos han reportado que la cantidad mínima de amidas necesaria para inhibir el crecimiento de los hongos Cladosporium sphaerospermum en placas TLC fue determinada de 0,1 a $5 \mu \mathrm{g}$ para $P$. tuberculatum y $5 \mu \mathrm{g}$ para P. hispidum (Navickiene et al. 2000). Asimismo, la actividad antifúngica de amidas contra $C$. sphaerospermum y C. cladosporioides fue determinada de 0,1 a $10 \mu \mathrm{g}$ para $P$. arboreum y 
Tabla 6. Inhibición en el crecimiento de Trichophyton rubrum, Microsporum canis y Microsporum gypseum por varias concentraciones de extracto de $\mathrm{CH}_{2} \mathrm{Cl}_{2}: \mathrm{MeOH}$ (2:1) de plantas in vitro de Piper tuberculatum.

\begin{tabular}{|c|c|c|c|}
\hline & & Inhibición (\%) & \\
\hline $\begin{array}{l}\text { Concentración } \\
(\mathrm{mg} / \mathrm{mL})\end{array}$ & T. rubrum & M. canis & M. gypseum \\
\hline 0,1 & 20 & 65 & 70 \\
\hline 0,5 & 100 & 100 & 80 \\
\hline 1,0 & 100 & 100 & 90 \\
\hline 1,5 & 100 & 100 & 95 \\
\hline
\end{tabular}

P. tuberculatum (Silva et al. 2002) y para ambas especies de hongos, las flavononas e hidroquinonas preniladas antifúngicas de $P$. crassinervium fue deteriminada de 1 a $10 \mu \mathrm{g}$ (Danelutte et al. 2003). Relacionado a estos trabajos, derivados del ácido benzoico en especies de Piper y su actividad antifúngica contra C. cladosporioides y C. sphaerospermum ha sido también reportada (Lago et al. 2004). Recientemente, la actividad antifúngica de siete nuevos cromenes de Peperomia villipetiola ha sido determinada para ambos hongos, donde la cantidad mínima requerida para la inhibición en el crecimiento del hongo en placas TLC fue $100 \mu \mathrm{g}$ (Malquichagua et al. 2005). En nuestro trabajo, la CIM contra T. rubrum, $M$. canis y $M$. gypseum fue $0,1 \mathrm{mg} / \mathrm{mL}$ o $100 \mu \mathrm{g} / \mathrm{mL}$. La inhibición en el crecimiento del hongo sugiere que compuestos como la piperidina, dihidropiridona y amidas isobutílicas de $P$. tuberculatum son compuestos de elevada actividad antifúngica e incluso más potentes que los cromenes de $P$. villipetiola. P. tuberculatum biosintetiza amidas con geometría cis en su cadena lateral, lo cual es una característica muy rara en la naturaleza (Shah et al. 1986; Alécio et al. 1998). Con certeza, el análisis de estos datos nos lleva a entender que la concentración adecuada para mostrar un determinado efecto depende de la especie vegetal utilizada y el tipo de extracto, donde la actividad sobre los microorganismos no se deba a la acción de un único principio activo sino al efecto sinergístico de varios de ellos que en la planta se encuentran en proporción minoritaria (Leatemia \& Isman 2004; Davicino et al. 2007)

Referente a los extractos de $\mathrm{CH}_{2} \mathrm{Cl}_{2}: \mathrm{MeOH}$ (2:1), obtenidos de plantas in vitro, la inhibición en el crecimiento fue $100 \%$ en concentraciones de 0,5 a $1,5 \mathrm{mg} / \mathrm{mL}$ pero solamente para $T$. rubrum y $M$. canis; para $M$. gypseum la inhibición en el crecimiento (70-95\%) fue ligeramente inferior (Tabla 6). Estos resultados abren la posibilidad de biosintetizar a gran escala los compuestos antifúngicos de $P$. tuberculatum mediante el establecimiento de suspensiones celulares, tal como se viene realizando en varias especies de importancia medicinal (Yeoman \& Yeoman 1996). En el caso de especies de Piper, en suspensiones celulares de P. cernuum fueron producidas las feniletilaminas dopamina $\mathrm{y}$ tiramina, y en $P$. crassinervium predominaron cuatro alkamidas, entre ellas la inédita 2,3,4-trimetoxy- $N$-metil-aristolactamano, en tanto, que en plantas adultas fenilpropanoides y ácidos benzoico prenilados, respectivamente, fueron los mayores compuestos detectados (Danelutte et al. 2005; Kato \& Furlán 2007). Adicionalmente, utilizando el método HPLC en fase reversa fueron detectadas las amidas antifúngicas e insecticidas dihidropiplartina, piplartina, $\Delta^{\alpha, \beta}$-dihidropiperina y pelitorina en plantas in vitro y callos de $P$. tuberculatum (Navickiene et al. 2003). Estos trabajos reafirman la importancia de varias modalidades de los cultivos in vitro en la biosíntesis de diversos metabolitos secundarios.

\section{Agradecimiento}

Este estudio fue financiado en parte con fondos de la Fundação de Amparo ao Pesquisa do Estado de São Paulo (FAPESP), São Paulo, Brasil.

\section{Literatura citada}

Alécio A.C., V.S. Bolzani, M.C.M. Young, M.J. Kato \& M. Furlan. 1998. Antifungal amides from leaves of Piper hispidum. J. Nat. Prod. 61: 637-639.

Araújo G.A., L.B. Galiza da Silva, D.R. da Silva, R. de Moraes Peixoto, et al. 2008. Dermatophytosis caused by Microsporum canis and Microsporum gypseum in free-living Bradypus variegatus (Schiz, 1825) in the state of Pernambuco, Brazil. Braz. J. Microbiol. 39: 508-510.

Brako L. \& J.L. Zarucchi. 1993. Catalogue of the Flowering Plants and Gymnosperms of Peru. Missouri Botanical Garden 45: 884-923.

Cano C., P. Bonilla, M. Roque \& J. Ruiz. 2008. Actividad antimicótica in vitro y metabolitos del aceite esencial de las hojas de Minthostachys mollis (muña). Rev. Peru Med. Exp. Salud pública. 25(3): 298-301.

Cos P., L. Maes, A. Vlietinck \& L. Pieters. 2008. Plant-derived leading compounds for chemotherapy of human immunodefiency virus (HIV) infection - an update (1998-2007). Planta Med. 2008: 1323-1337.

Dabur R., H. Singh, A.K. Chhillar, M. Ali \& G.L. Sharma. 2004. Antifungal potential of Indian medicinal plants. Fitoterapia 75(3-4): 389-391.

Danelutte A.P., J.H.G. Lago, M.C.M. Young \& M.J. Kato. 2003. Antifungal flavanones and prenylated hydroquinones from Piper crassinervium Kunth. Phytochemistry 64: 555-559.

Danelutte A.P., M. Costantin, G.E. Delgado, R. Braz-Filho \& M.J. Kato. 2005. Divergence of secondary metabolism in cell suspensión cultures and differentiated plants of Piper cernuum and P. crassinervium. J. Braz. Chem. Soc. 16 No 6B: 1425-1430.

Davicino R., M.A. Mattar, Y.A. Casali, S.G. Correa, E.M. Pettenati \& B. Micalizzi. 2007. Actividad antifúngica de extractos de plantas usadas en medicina popular en Argentina. Rev. peru. biol. 14(2): 247-251.

Fenner R., M. Sortino, S.M. Rates, R. Dall'Agnol, A. Ferraz, et al. 2005. Antifungal activity of some Brazilian Hypericum species. Phytomedicine 12(3): 236-240.

Grayer R.J. \& J.B. Harborne. 1994. A survey of antifungal compounds from higher plants. 1982-1993. Phytochemistry 37(1): 19-42.

Gibbons S. 2008. Phytochemicals for bacterial resistance-strengths, weaknesses and opportunities. Planta Med. 74: 594-602.

Hernández T., M. Canales, B. Teran, O. Avila, et al. 2007. Antimicrobial activity of the essential oil and extracts of Cordia curassavica (Boraginaceae). J. Ethnopharm. 111: 137-141.

Homans A.L. \& A. Fuchs. 1970. Direct bioautography on thin-layer chromatograms as a method for detecting fungitoxic substances. J. Chromatograph. 51: 327-329.

Kato M.J. \& M. Furlan. 2007. Chemistry and evolution of the Piperaceae. Pure Appl. Chem. 79(4): 529-538.

Koroishi A.M., S.R. Foss, D.A.G. Cortez, T. Ueda-Nakamura, C. Vataru \& B.P. Dias Filho. 2008. In vitro antifungal activity of extracts and neolignans from Piper regnellii against dermatophytes. J. Ethnopharm. 117: 270-277.

Lago J.G.L., C.S. Ramos, C.C.D. Casanova, A. de A. Morandim, et al. 2004. Benzoic acid derivates from Piper species and their fungitoxic activity against Cladosporium cladosporioides and C. sphaerospermum. J. Nat. Prod. 67: $1783-1788$ 
Leatemia J. \& B. Isman. 2004. Toxicity and antifeedant activity of crude seed extracts of Annona squamosa (Annonaceae) against epidopteran pests and natural enemies. Int. J. Trop. Insect Sci. 24(1): 150-158.

Levy B.H.D., J.D.L. Fedullo, S.H.R. Corrêa, R.H.F. Teixeira \& S. Dall' Acqua. 2006. Isolation of Microsporum gypseum from tha haircoat of health wild felids kept in captivity in Brazil. Braz. J. Microbiol. 37: 148-152.

Malquichagua K.J., G.E. Delgado, L. Ripalda, M.C.M. Young \& M.J. Kato. 2005. Chromenes of polyketide origin from Peperomia villipetiola. Phytochemistry 66: 573-579.

Martins R.C.C., L.R. Latorre, P. Sartorelli \& M.J. Kato. 2000. Phenylpropanoids and tetrahydrofuran lignans from Piper solmsianum. Phytochemistry 55: 843-846.

Miyakado M., I. Nakayama \& N. Ohno. 1989. Insecticides of plant origin. En: J.T. Arnason, B.J.R. Philogène \& P. Morand. (Eds). ACS Symposium Series Am. Chem. Soc. Washington, DC. p. $387-390$

Murashige T. \& F. Skoog. 1962. A revised medium for rapid growth and bioassays with tobacco tissue cultures. Physiol. Plant. 15: 473-497.

Navarro-García V.M., A. Gonzalez, M. Fuentes, M. Avilés, M.Y. Ríos, G. Zepeda \& M.G. Rojas. 2003. Antifungal activities of nine traditional Mexican medicinal plants. J. Ethnopharmacol. 87(1): 85-88.

Navickiene H.M.D., M.J. Kato, V. da S. Bolzani, M.C.M. Young, G.E. Delgado \& M. Furlan. 1999. Amidas antifúngicas isoladas de Piper tuberculatum (Piperaceae). 23a . Reunião Anual da Sociedade Brasileira de Química, Poços de Caldas, MG, Brasil. PN-096. p. 96.

Navickiene H.M.D., A.C. Alécio, M.J. Kato, V. da S. Bolzani, M.C.M. Young, A.J. Cavalheiro \& M. Furlan. 2000. Antifungal amides from Piper hispidum and Piper tuberculatum. Phytochemistry 55: 621-626.

Navickiene H.M.D., V. da S. Bolzani, M.J. Kato, A.M.S. Pereira, B.W. Bertoni, S. Castro \& M. Furlan. 2003. Quantitative determination of anti-fungal and insecticide amides in adult plants, plantlets and callus from Piper tuberculatum by reverse-phase high-performance liquid chromatography. Phytochem. Analysis 14: 281-284.

Orjala J., A.D. Wright, H. Behrends, G. Folker \& O. Sticher. 1994. Cytotoxic and antibacterial dihydrochalcones from Piper aduncum. J. Nat. Prod. 57: 18-26.
Osbourn A.E. 1996. Preformed antimicrobial compounds and plant defense against fungal attack. Plant Cell 8: 1821-1831.

Pessini G.L., B.P. Dias Filho, C. Vataru \& D.A. Garcia. 2005. Antifungal activity of the extracts and neolignan from Piper regnellii (Miq.) C.DC. var. pallescens (C. DC.) Yunck. J. Braz. Chem. Soc. 16(6A): 1130-1133.

Rojas R., B. Bustamante, J. Bauer, I. Fernández, J. Albán \& O. Lock. 2008. Antimicrobial activity of selected Peruvian medicinal plants. J. Ethnopharm. 88: 199-204.

Shah S., A.K. Kalla \& K.L. Dhar. 1986. A cinnamoyl pyrrolidine amide from Piper peepuloides. Phytochemistry 25: $1997-$ 1998.

Shamim S., S. Waseemuddin Ahmed \& I. Azhar. 2004. Antifungal activity of Allium, Aloe, and Solanum species. Pharm. Biol. 42: 491-498.

Silva R.V., H.M.D. Navickiene, M.J. Kato, V. da S. Bolzani, C.I. Méda M.C.M. Young \& M. Furlan. 2002. Antifungal amides from Piper arboreum and Piper tuberculatum. Phytochemistry 59: 521-527.

Soberón, G.V., C. Rojas, J. Saavedra, M.J. Kato \& G.E. Delgado. 2006. Acción biocida de plantas de Piper tuberculatum Jacq. sobre Diatraea saccharalis (Lepidóptera, Pyralidae. Rev. peru. biol. 13(1): 107-112.

Tebbs M.C. 1989. Revision of Piper (Piperaceae) in the New World. 1. Review of characters and taxonomy of Piper section Macrostachys. Bull. Br. Mus. Nat. Hist. (Bot). 19: 117-158.

Trakranrungsie N., A. Chatchawanchonteera \& W. Khunkitti. 2008. Etnoveterinary study for antidermatophytic activity of Piper betle, Alpinia galanga and Allium ascalonicum extracts in vitro. Res. Veter. Sci. 84: 80-84.

Trelease W. 1936. 40. Piperaceae. En: Macbride, J.F. Flora of Peru. Field Museum of Natural History, Botanical Series, Chicago, USA. 253 p.

Yeoman M.M. \& C.L. Yeoman. 1996. Manipulating secondary metabolism in cultured plant cells. Tansley Review No 90. New Phytol. 134: 553-569.

Yuncker T.G. 1973. The Piperaceae of Brazil. II. Piper - Group V; Ottonia, Pothomorphe, Sarcorhachis. Hoehnea 3: 29-284.

Zhang J.D., Y.B. Cao, Z. Xu, H.H. Sun, M.M. An, L. Yan, H.S. Chen, R.H. Gao, Y. Wang, X.M. Jia \& Y.Y. Jiang. 2005. In vitro and in vivo antifungal activities of the eight steroid saponins from Tribulus terrrestris $\mathrm{L}$. with potent activity against fluconazole-resistant fungal pathogens. Biol. Pharm. Bull. 28(12): 2211-2215. 